Cahiers $d u$ MONDE RUSSE

\section{Cahiers du monde russe}

Russie - Empire russe - Union soviétique et États indépendants

$50 / 1 \mid 2009$

Écrits personnels. Russie XVIII ${ }^{\mathrm{e}} \mathrm{XX} \mathrm{X}^{\mathrm{e}}$ siècles

\title{
L'usage du français et du russe dans les journaux
}

féminins

XVIII $>^{\mathrm{e}}-$ premier tiers du XIX $>^{\mathrm{e}}$ siècle

\section{Elena Greanaja}

\section{OpenEdition}

1 Journals

Édition électronique

URL : https://journals.openedition.org/monderusse/9148

DOI : 10.4000/monderusse. 9148

ISSN : $1777-5388$

Éditeur

Éditions de l'EHESS

Édition imprimée

Date de publication : 31 mars 2009

Pagination : 21-32

ISBN : 978-2-7132-2259-7

ISSN : $1252-6576$

Référence électronique

Elena Greanaja, "L'usage du français et du russe dans les journaux féminins », Cahiers du monde russe [En ligne], 50/1 | 2009, mis en ligne le 01 janvier 2009, consulté le 03 septembre 2022. URL : http://journals.openedition.org/monderusse/9148; DOl : https://doi.org/10.4000/monderusse.9148 


\title{
L'USAGE DU FRANÇAIS ET DU RUSSE DANS LES JOURNAUX FÉMININS
}

\author{
XVIII ${ }^{\mathrm{e}}$-premier tiers du XIX ${ }^{\mathrm{e}}$ siècle
}

Les premiers journaux personnels, qui apparaissent en Russie dès la fin $\mathrm{du}$ $\mathrm{XVII}^{\mathrm{e}}$ siècle, sont rédigés par des hommes qui les tiennent, pour la plupart, en russe ${ }^{1}$. Les premiers journaux connus de femmes russes, nobles, datent de la seconde moitié du XVIII ${ }^{\mathrm{e}}$ siècle, époque de la diffusion, de plus en plus intense dans la société aristocratique de la Russie, de la langue et de la culture françaises, et sont rédigés en français. À cette époque, à côté des journaux tenus en russe, les hommes rédigent, eux aussi, des journaux en français, et il existe quelques cas où les auteurs recourent aux trois langues : le français, l'allemand et le russe ${ }^{2}$.

Cependant, à en juger d'après les journaux publiés du XVIII et du premier tiers du XIX ${ }^{\mathrm{e}}$ siècles $^{3}$ et le corpus des textes inédits de la même époque conservés dans les archives de Moscou et Saint-Pétersbourg ${ }^{4}$, le mélange des langues, en premier lieu

1. Voir A.G. Tartakovskij, Russkaja memuaristika XVIII-pervoj poloviny XIX v. [Mémoires et journaux personnels russes du XVIII ${ }^{\mathrm{e}}$-première moitié du XIXe siècle], M. : Nauka, 1991.

2. Le journal des trois frères Pavel, Petr et Aleksandr Demidov (commencé en 1758), signalé par W. Berelowitch dans son article «Les récits des voyageurs russes en France dans la seconde moitié du XVIII ${ }^{e}$ siècle », in G. Dulac, D. Taurisson, M. Piha et M. Reverseau, éds., La culture française et les archives russes: Une image de l'Europe au XVIII' siècle, FerneyVoltaire, 2004, p. 11, et celui d'Alexej Bobrinskij, publié dans S. Kozlov, Russkij puteščestvennik epohi Prosveščenija [Le Voyageur russe à l'époque des Lumières], t. 1., SPb. : Istoričeskaja illustracija, 2003, p. 356-447.

3. Voir Istorija dorevoljucionnoj Rossii $v$ dnevnikah $i$ vospominanijah: Annotirovannyj ukazatel' knig i publikacij v žurnalah [Histoire de la Russie d'avant la révolution d'après les journaux et les mémoires : Index annoté des livres et des publications dans des revues], sous la direction de P.A. Zajončkovskij, t. 1, XVe-XVIII ${ }^{\mathrm{e}}$ siècles, M. : Kniga, 1976 ; t. 2, partie 1, 1801-1856, M. : Kniga, 1977.

4. Ces journaux ont fait l'objet d'une étude dans le cadre du Programme international de coopération scientifique, en collaboration avec Catherine Viollet (Institut des textes et manuscrits modernes, CNRS). 
française et russe, est plus fréquent dans les journaux des femmes russes, surtout à partir des années $1820^{5}$ (tandis que l'alternance des langues est pratiquement absente dans les journaux masculins du premier tiers du XIX siècle). Ce fait correspond par ailleurs à la pratique épistolaire féminine de cette époque ${ }^{6}$.

L'objectif de cet article est de montrer comment et dans quelle mesure l'alternance des langues influe sur l'expression personnelle et la construction du moi, sur la «manière d'être $»^{7}$, et quelles sont les fonctions respectives du français et du russe dans les journaux personnels féminins de l'époque en question. Les journaux féminins de ce type, alternant le français et le russe, ne sont pas nombreux mais constituent cependant une partie considérable du corpus étudié. Parmi la dizaine d'auteurs de textes publiés, quatre ont recours aux deux langues (Anna Kern, Anastasija Kolečickaja, Anna Olenina et Varvara Šeremeteva), et, sur la quarantaine d'auteurs de manuscrits, six sont dans ce cas (Natal'ja Golicyna, Marija Bahmeteva, Elisaveta Šahovskaja, Marija Tolstaja, Ekaterina Vadkovskaja, Varvara Suhotina). Un journal, appartenant à Natal'ja Stroganova, rédigé entièrement en français, fait aussi l'objet de notre étude car elle y relate les mêmes événements dans un autre texte, rédigé en russe. Tous les auteurs des journaux sont les représentantes de la noblesse ${ }^{8}$.

\section{Relation d'un voyage en français et en russe}

L'un des premiers journaux féminins tenus en français est celui de la baronne Natal'ja Mihajlovna Stroganova (née princesse Beloselskaja ; 1743-1819), sœur du prince Aleksandr Beloselskij, diplomate russe et écrivain français. Une copie, de cent-dix pages, en est conservée, apparemment établie d'après les notes prises en cours de route. La baronne voyage en Europe en 1780-1783. Le journal est visiblement destiné à circuler ensuite dans l'entourage familial, où le français est bien maîtrisé : il est dédié aux sœurs de l'auteur. La baronne Stroganova séjourne à Berlin, Dresde, Spa, Paris, Londres et Vienne.

L'auteur du journal donne en premier lieu une chronique de son voyage, un compte rendu détaillé du parcours, en indiquant les lieux où l'on s'arrête pour changer de chevaux, se restaurer, se reposer. Cette chronique s'appuie sur des

5. Je renvoie à notre ouvrage «Si tu lis jamais ce journal...», Diaristes russes francophones. 1780-1854, E. Gretchanaia, C. Viollet, éds., P. : CNRS Éditions, 2008.

6. I.A. Paperno, «O dvujazyčnoj perepiske puškinskoj epohi» [De la correspondance bilingue à l'époque de Puškin], Učenye zapiski tartuskogo universiteta, vyp. 358, Tartu, 1975, p. 149.

7. Voir Michel Braud, La forme des jours : Pour une poétique du journal personnel, P. : Seuil, 2006, p. 197-200.

8. Des extraits des journaux de Natal’ja Stroganova, Natal’ja Golicyna, Ekaterina Vadkovskaja, Marija Bahmeteva, Marija Tolstaja, Elizaveta Šahovskaja sont publiés dans «Si tu lis jamais ce journal... ». 
guides de voyage français qui serviront pendant longtemps de repères aux auteurs des journaux ${ }^{9}$.

La langue française permet de peindre un tableau authentique de la vie européenne, mais aussi de se divertir lors des déplacements. La baronne Stroganova dit avoir composé une comédie destinée à être jouée en route par les enfants qui voyagent avec elle et ses compagnons ${ }^{10}$. Plus tard, ses petites compositions en français dans le genre de la littérature de salon vont figurer dans les albums de « l'Académie Strogonovienne » [sic], terme désignant les réunions de la société mondaine qui avaient lieu dans sa maison au début du XIX $\mathrm{X}^{\mathrm{e}}$ siècle ${ }^{11}$.

Par ailleurs, le journal-chronique de Natal'ja Stroganova contient quelques échappées dans le monde intérieur de l'auteur. La représentation de ce monde se plie généralement aux canons de la littérature sentimentale : il est imprégné de sensibilité, voire même d'une certaine tristesse :

Je passe sous silence le nom des postes où il n'est rien arrivé de remarquable, sinon que les chevaux étoient mauvais et que les Voyageurs étoient tristes à cause de la séparation des personnes avec les quelles ils se sont séparés surtout moi, ayant le caractere fort tendre, j'étois affligée à ne point savoir retenir mes larmes ; j'ai quitté mes sœurs pour la premiere fois de ma vie pour un terme aussi long et rien n'auroit pu m'en consoler si je ne me trouvois avec ma meilleure amie la C.[omtesse] S.[altykova]. ${ }^{12}$

L'auteur évite cependant de décrire en détail ses états d'âme, qu'elle dit ne communiquer qu'à sa parente et amie avec laquelle elle voyage :

[...] après le diner nous sommes arrivés à Memel, j'étois fort triste à cause des différentes idées que j'ai eu avec ma chère amie que j'aime de tout mon cœur ${ }^{13}$.

L'empreinte de cette réserve, formée à la croisée de la culture aristocratique française et du « silence » traditionnel de la femme russe, marquera pour longtemps les journaux féminins russes en langue française.

Il est à noter que de 1760 jusqu'en 1815 (date de la mort de son fils), la baronne Stroganova poursuit en russe la chronique de sa famille commencée par son père en 1724 et continuée après sa mort par sa mère (décédée à son tour en 1760). Dans

9. Voir, pour les voyages en France, Wladimir Berelowitch, « La France dans le "grand tour" des nobles russes au cours de la seconde moitié du XVIII siècle », Cahiers du Monde russe et soviétique, 34 (1-2), 1993, p. 195-209.

10. «Si tu lis jamais ce journal... »,p. 72 .

11. Voir M.K. Azadovskij, «Iz materialov "Strogonovskoj Akademii”. Neizdannye proizvedenija Ksav'e de Mestra i Zianaidy Volkonskoj» [Matériaux de "l'Académie Strogonovienne ». Euvres inédites de Xavier de Maistre et Zinaïda Volkonskaja], Literaturnoe nasledstvo, 33-34, 1939, p. 195-214.

12. «Si tu lis jamais ce journal... », p. 71-72. Ici et plus loin, l'orthographe des diaristes est conservée telle que dans l'original. La comtesse Darja Petrovna Saltykova (née comtesse Černyševa ; 1738-1802) était la cousine de la baronne Stroganova.

13. Ibid., p. 73. 
cette chronique russe, la baronne se montre parfaitement conforme à ce genre. Elle enregistre de manière extrêmement laconique les événements essentiels qui ont lieu dans sa famille : morts, naissances, avancements des hommes dans leur carrière, déplacements. Le compte rendu de son voyage en Europe n'y occupe que quelques lignes et se présente ainsi :

В 178011 июля выехали ис Петербурха в чужие краи на два года Г[раф] Иван Петрович Салтыков ${ }^{14}$ з женой, 3 двумя дочерми, а я с сыном. 26 июля приехали мы в Берлин. 6 августа из Берлина уехали и 8 числа августа в ночь приехали в Дрезден, где брат князь Александр Михаилыч министром. 13 августа выехали из Дрездена. 28 августа приехали в Спа. Г[рафине] Дарье Петровне надо была лечитца [Le 11 juillet 1780 nous sommes partis de Pétersbourg dans les pays étrangers pour deux ans, le Comte Ivan Petrovič Saltykov avec sa femme et ses deux filles, et moi avec mon fils. Le 26 juillet, nous sommes arrivés à Berlin. Le 6 août, nous sommes partis de Berlin et le 8 août, dans la nuit, nous sommes arrivés à Dresde où mon frère, le prince Aleksandr Mihajlovič, est ministre. Le 13 août, nous sommes partis de Dresde. Le 28 août, nous sommes arrivés à Spa. La Comtesse Darja Petrovna avait besoin d'être soignée.$^{15}$

Le séjour à Paris, où la baronne arrive le 8 octobre 1780 et reste jusqu'en avril 1783, tient une place considérable dans son journal tenu en français. Elle raconte comment elle s'y fait peindre par Greuze, qu'elle entend l'abbé Delille lire un chant de son poème Les Jardins, visite la demeure du maréchal de Biron, de la maréchale de Luxembourg, de la duchesse de La Vallière et de Mme Necker, centres de la sociabilité parisienne, dîne chez la duchesse de Chartres et déjeune chez l'abbé Raynal, se promène dans la ville avec la comtesse Šuvalova, épouse du comte Andrej Šuvalov, poète français, et avec la princesse Ekaterina Daškova. À Versailles, la baronne est reçue par Marie-Antoinette, qui lui fait visiter ses appartements.

En revanche, dans la chronique russe, Natal'ja Stroganova note seulement à propos de son séjour à Paris et la fin de son voyage :

[...] приехали в Париж 8 октября. В Париже жили очень весело. Я с Г[рафиней] Брюсовой подружилась. Ездили в Лондон на 3 месяца. В апреле 1783 выехали из Парижа. Приехали в Россию 25 июня того же года [...nous sommes arrivés à Paris le 8 octobre. Nous avons vécu à Paris très joyeusement. Je suis devenue amie de la C[omtesse] Bruce. Nous sommes allés à Londres pour trois mois. En avril 1783, nous sommes partis de Paris. Nous sommes arrivés en Russie le 25 juin de la même année]. ${ }^{16}$

En comparant les deux relations, on peut donc constater que, dans le texte en français, l'auteur se sent plus libre et peut-être plus portée à donner quelques détails sur la vie quotidienne et les préoccupations qui lui sont liées, à préciser les avantages et

14. Le comte Ivan Petrovitch Saltykov (1730-1805), général en chef.

15. K.A. Pisarenko, «Iz semejnoj hroniki roda Stroganovyh» [Chronique familiale des Stroganov], Rossijskij arhiv, M., 2005, p. 75 (ici et plus loin, c'est moi qui traduis).

16. Ibid., p. 76. 
inconvénients des lieux parcourus, à présenter parfois, même si c'est d'une manière réservée, son paysage intérieur, à faire part de ses affections et à se plaindre des ennuis de santé.

\section{Recours « technique » à la langue russe}

Dans les journaux féminins tenus en français, la langue russe peut servir en premier lieu pour transcrire les noms propres, les toponymes ou des notions ayant trait à la religion orthodoxe. On trouve ce procédé dans les journaux de la princesse Natal'ja Golicyna, de Marija Bahmeteva et de Varvara Suhotina. Ainsi la princesse Natal'ja Petrovna Golicyna (née comtesse Černyševa ; 174417-1837) écrit-elle dans son journal de 1781(?)-1783, qui commence par une chronique rétrospective :

Je fu promise le $\underline{15}$ de Janvier de lan 1766 au Prince Владимир Борисович Голицын [Vladimir Borisovič Golicyn] qui étoit colonel dans le Régiment de Пермской пехотной, обручали нас перстнями во дворце [d'infanterie de Perm, on nous mit les bagues de fiançailles au palais]. ${ }^{18}$

Marija Bahmeteva, une jeune fille née vers la fin des années 1780, inscrit, dans son journal tenu à Pétersbourg en 1805-1807, les noms propres en russe, surtout quand ils se composent du prénom et du patronyme (« Дмитрий Моисеевич » [Dmitrij Moiseevič]), de même que les titres des spectacles russes («Князь-невидимка » [Le prince invisible]) Si le mot russe botvinia [soupe à la betterave] apparaît dans son journal transcrit en caractères latins, sa bonne connaissance du russe est attestée par le fait qu'elle lit et traduit du russe en italien les extraits du récit d'Aleksandra Hvostova Kamin [La Cheminée, 1796] ${ }^{19}$.

Plus tard, dans le journal de voyage de 1828 à l'intérieur de la Russie de Varvara Sergejevna Suhotina (née Domašneva ; vers 1795-après 1856), ce sont en premier lieu des réalités liées à la religion orthodoxe qui invitent à passer à la langue russe : « L'église du Couvent est grande, d'une architecture gothique, ressemblant à notre paroisse de Moscou, Петра и Певла. Тут есть явленный образ Володимерской божией матери, к которому мы прикладывались [Pierre et Paul. Il y a là une icône miraculeuse de Notre-Dame de Vladimir que nous avons embrassée]. Il y a peu de moines dans ce couvent... $\gg^{20}$. La relation du voyage de Varvara Suhotina, ainsi que les réflexions concernant ses lectures en français, sont consignées dans cette langue.

17. Le journal de la princesse Golicyna permet de rectifier la date convenue de sa naissance (1741) : elle est née à Berlin deux ans avant le départ de sa famille pour Londres (1746), il s'agit donc de l'année 1744.

18. « Si tu lis jamais ce journal... »,p. 82 .

19. Ibid., p. 175.

20. V.S. Suhotina, [Journal] (autographe), RGADA (Rossijskij Gosudarstvennyj arhiv drevnih aktov - Archives d'État des actes anciens de Russie], f. 1280, op. 1, d. 136, 1. 4vº. 


\section{Le choix de la langue dans les journaux bilingues}

Durant le premier tiers du XIX ${ }^{\mathrm{e}}$ siècle, se poursuit la formation de la langue littéraire russe. Le rôle des femmes dans cette situation langagière est particulièrement important : en effet, «le goût de la femme mondaine» constituait, depuis la réforme du russe par Nikolaj Karamzin, l'un des critères de la langue correcte, tant écrite que parlée ${ }^{21}$.

Si le français reste la langue principale des journaux personnels des femmes russes nobles, vers les années 1820 apparaissent des journaux qui peuvent être qualifiés de bilingues, où des passages en russe tiennent une place considérable - phénomène lié au développement de la littérature russe et de la conscience nationale.

Les diaristes manifestent alors leur penchant pour la langue maternelle. Ainsi Varvara Petrovna Šeremeteva (née Almazova ; 1786-1857), qui tient un journal par lettres (1825-1826) lors de son voyage de Moscou à Pétersbourg, exprime en russe son mécontentement d'écrire en français, usage qui ne la satisfait apparemment pas :

я не знаю, отчего я все объясняюсь на французском диалекте, пишу весьма худо и верите ли, что иногда останавливаюсь, не знаю, как написать ... [je ne sais pourquoi je m'exprime toujours en dialecte français, j'écris très mal et, le croirez-vous, parfois je m'arrête, ne sachant comment écrire]. ${ }^{22}$

Le journal bilingue le plus connu de cette époque est celui qu'Anna Kern tient en province, en $1820^{23}$. Représentante de la petite noblesse de province, Anna Kern maîtrise bien sa langue maternelle qui lui est aussi familière que le français, et porte l'empreinte évidente des sentimentalistes russes. L'auteur traduit avec aisance certains passages qui lui plaisent particulièrement dans l'ouvrage de Mme de Staël De l'Allemagne, et parle de ses propres sentiments en recourant à un russe livresque.

Dans le journal de 1820 d'Anastasija Ivanovna Kolečickaja (née Lykošina; 1800-1871), c'est le russe qui prédomine. L'auteur passe généralement au français lors de ses digressions concernant les devoirs et la vertu des femmes, ses lectures d'ouvrages philosophiques, lors de réflexions religieuses et d'épanchements sentimentaux au sein de la nature 24 .

21. Voir V.V. Vinogradov, Očerki russkogo literaturnogo jazyka XVII-XIX vekov [Essais sur la langue littéraire russe des XVII ${ }^{\mathrm{e}}$-XIXe siècles], M. : Vysšaja škola, 1982 (1 re éd. 1934), p. 194 ; B.A. Uspenskij, Iz istorii russkogo literaturnogo jazyka XVIII-načala XIX veka : Jazykovaja programma Karamzina i ee istoričeskie korni [De l'histoire de la langue littéraire russe du XVIII ${ }^{\mathrm{e}}$-début du XIXe siècle : Le programme langagier de Karamzin et ses origines historiques], M. : izdatel'stvo Moskovskogo universiteta, 1985, p. 154-155.

22. V.P. Šeremeteva, [Journal] (copie dactylographiée du début du $\mathrm{XX}^{\mathrm{e}}$ siècle), RGADA, f. 1287, op. 1, d. 5160, 1.38. Ce journal est publié en traduction russe : V.P. Šeremeteva, Dnevnik [Journal], M. : typographie de Synode, 1916.

23. A.P. Kern (Markova-Vinogradskaja), Vospomonanija, Dnevniki, Perepiska [Souvenirs, Journaux, Correspondance], M. : Hudlit, 1974.

24. A.I. Kolečickaja, « Moi zapiski ot 1820 goda » [Mes notes de l'an 1820], in E.E. Liamina et E.E. Pasternak, éds., Lica: Biografičeskij al'manah [Visages: almanach biographique], M. : Abrahm Reutblat; SPb : Phenix ; Atheneum, n 6, 1995,p. 277-341. 
Dans le journal connu d'Anna Aleksejevna Olenina (1808-1888), rédigé en 1828, le russe, langue qu'elle maîtrise fort bien, l'emporte également. Commençant en russe, elle parle d'un ton moqueur de ses états d'âme de jeune fille amoureuse. Ensuite, la tonalité change : c'est en français qu'elle cite le poème de Lamartine « L'isolement», et poursuit ses réflexions en se projetant dans la situation livresque :

Voilà la vie de ceux qui ont trop senti dans leur jeunesse, voilà la vie de celle, dont la main trace ces lignes. À peine à l'âge de vingt ans, et elle a cessé déjà de jouir. Sans un seul vrai malheur et sentant avec toute la chaleur d'une âme enthousiaste, tout le bonheur d'une vie passée avec des êtres adorés, sans avoir éprouvé encore aucune rigueur du sort elle est « comme la feuille flétrie $»^{25}$, car la raison lui a ôté d'une main barbare toutes les illusions! sans fortune, sans beauté elle attache pour un jour, un mois, mais jamais pour la vie. La jeunesse s'écoule, le bonheur fuit pour ne revenir jamais! ... Ah, pardonnez-lui alors de dire : « Emportez-moi, comme elle, orageux Aquilon ! $»^{26}$

En présentant dans son journal sa vie comme un roman, elle lui donne un titre dans le goût du XVIII' siècle :

Il faut avouer que le roman est riche en caractères variés... Mais commençons. Comment appeler ce roman ? pensons... Le voilà trouvé !

Les inconséquences ou pardonnez à l'Amour. ${ }^{27}$

Le français est également utilisé pour esquisser des portraits de son entourage, pour noter conversations mondaines et nouvelles politiques.

Les journaux de la princesse Elizaveta Šahovskaja et de la comtesse Marija Tolstaja représentent un cas quelque peu différent. C'est bien le français qui leur est apparemment la langue la plus familière.

«Un seul être vous manque, et tout est dépeuplé » : ce vers, également tiré de «L'Isolement » de Lamartine, règle la tonalité du journal de 1822-1824 d'Elizaveta Aleksandrovna Šahovskaja (née Muhanova; 1803-1836) ${ }^{28}$. À cette époque, elle n'est pas encore mariée et son journal est imprégné de son amour pour le prince Valentin Šahovskoj, son futur mari, souvent absent. Elizaveta conçoit l'écriture de son journal comme une sorte de projet littéraire et n'est guère satisfaite de la qualité de son texte. Cependant elle tient à son journal, refuse de le détruire aux moments de déception et y revient comme à son œuvre : « ... et me voilà la plume en main pour noter et mon bonheur et mes faiblesses ${ }^{29}$. Dans ses longues entrées, il y a très

25. «Et moi, je suis semblable à une feuille, Emportez-moi comme elle, orageux Aquilon », vers de « L'Isolement » de Lamartine.

26. A.A. Olenina, Dnevnik: Vospominanija [Journal. Souvenirs], SPb.: Akademičeskij proekt, 1999, p. 57.

27. Ibid., p. 65-66.

28. E.A. Šahovskaja, [Journal] (autographe), RGB (Rossijskaja gosudarstvennaja biblioteka Bibliothèque de l'État russe), f. 336/2, carton $47, \mathrm{n}^{\circ} 3, \mathrm{f}^{\circ} 16 \mathrm{v}$.

29. Ibid., $\mathrm{n}^{\circ} 7, \mathrm{f}^{\circ} 1 \mathrm{v}$. 
peu de ratures : elle préfère un style orienté vers la spontanéité, la négligence et la liberté d'expression héritées de la pratique épistolaire française.

Lorsqu'elle relate en français l'histoire de son amour pour Valentin, son récit prend les allures d'un roman français :

A l'âge de seize ans, j'ai commencé à aller dans le monde, et au bal de la $\mathrm{P}[\mathrm{rin}]$ cesse Bobrinsky $\underline{\mathrm{n}[\mathrm{ou}] \mathrm{s}}$ avons débuté ensemble. J'ai fait connaissance avec plusieurs jeunes gens, entre autres avec un superbe homme, qui me fit la cour, m'aima je l'appris par un cousin très dévoué, et la tête exaltée d'une personne de 16 ans fut montée par de belles espérances de l'épouser dans deux ans, je ne pensais plus à mon ami d'enfance, lui aussi il fut occupé d'une jolie personne, après ces petits sentimens se sont calmés et d'autres nous ont occupés chacun avoit son objet, et tous deux nous étions trompées. Enfin nous sommes revenus l'un à l'autre, il m'a beaucoup aimé, et nous étions excessivement liés jusqu'à ce jour, qu'il m'a demandé le 21 Mai 1821 que Si je pouvois lui donner la parole d'être à lui dans deux ans, je lui répondis que je ne pouvois le lui promettre pour si longtems, car je ne pouvois pas être sûre de lui - il me prit la main et me la serra en priant de penser à lui. Il alla au jardin pour graver sur un arbre, en mémoire de ce jour, cette date. ${ }^{30}$

Dans le journal d'Elizaveta Šahovskaja, ainsi que dans celui de la comtesse Marija Jur’ jevna Tolstaja (née princesse Obolenskaja ; 1802-après 1847), tenu en 18211823, le français reste la langue favorite et habituelle de la chronique familiale et mondaine, de la description parfois romanesque de leur vie. Les passages dans lesquels Marija Tolstaja rapporte les conversations qu'elle a tenues avec ses cavaliers lors d'innombrables bals ressemblent à des dialogues de roman ${ }^{31}$.

Le français forme et alimente le discours religieux d'Elizaveta Šahovskaja, qui tient une grande place dans ses journaux et s'appuie sur des textes spirituels français. Dans un cahier de citations de 1823, elle transcrit des passages du texte intitulé Des vraies amitiés qui se situe dans la troisième partie de l'Introduction à la vie dévote de saint François de Sales (1609 ; les dernières éditions qu'a pu lire Elizaveta parurent en 1820 et 1822), ainsi que des extraits des lettres spirituelles de Fénelon (il y avait deux éditions de ses œuvres à cette époque : Euvres complètes, en 10 volumes, 1810, et Euvres choisies, en 6 volumes, 1821) et de La consolation du chrétien, ou Motif de confiance en Dieu dans les diverses circonstances de la vie de l'abbé Roissard (publié en 1775 ; Elizaveta a pu lire l'édition de 1822 ou de 1818). Cette lecture est alors appliquée à sa propre expérience :

Vois-tu, cher ami [elle s'adresse à Valentin Šahovskoj], comme St François de Sales justifie notre attachement [...] Quel est le lien de notre amitié ? N'est-ce pas le désir de nous perfectionner mutuellement [...] Je te verois aujourd'hui et t'engagerai à lire François de Sales ${ }^{32}$.

30. « Si tu lis jamais ce journal... », p. 238.

31. Voir ibid., p. 247-248.

32. E.A. Šahovskaja, [Cahiers de citations], autographe, RGB, 336/II, carton 70, $\mathrm{n}^{\circ} 4, \mathrm{f}^{\circ} 2$. 
Dans son journal de 1824, elle mentionne, parmi les livres lus, d'autres ouvrages catholiques : Le combat spirituel de Laurent Scrupoli (XVI e siècle), traduit en français ${ }^{33}$, L'Évangile médité ${ }^{34}$, les œuvres de Massillon. Après son mariage, elle note dans son journal de 1825 qu'elle lit avec son mari chaque matin L'Imitation de Jésus-Christ ${ }^{35}$.

Le recours à la langue russe apparaît dans les journaux d'Elizaveta Šahovskaja et de Marija Tolstaja comme un choix volontaire, qui devient objet de réflexions.

Elizaveta Šahovskaja connaît les œuvres de Žukovskij, de Puškin, mais aussi d'Aleksandr Bestužev - défenseur, dans les revues de son temps, de la langue russe - , avec lequel elle a l'occasion de parler en mars 1823 :

Бестужев [Bestužev] qui est parti aujourd'hui, a dîné chez nous, il est rempli d'esprit ; nous avons beaucoup causé sur la littérature russe, qu'il connaît à fond ; il est si rare de trouver un jeune homme qui s'occupe réellement de sa langue paternelle. ${ }^{36}$

Elizaveta exprime le souhait de maîtriser sa langue maternelle, se prescrit la lecture quotidienne de l'Évangile, en russe et en slavon :

[...] я всегда начинаю свой день читая некоторое время книгу, которая может способствовать мне к познанию самого себя; возвратясь из той деревни, я назначила читать каждый день пять глав из Евангелии; для того что во время нашей бытности здесь я непременно хочу прочесть весь новый Завет один раз по русски, а другой по славянски; я люблю свой отечественый язык и желаю утвердиться в нем; и мне приятно читать Евангелие на своем языке.» [[...] je commence toujours ma journée en lisant quelque temps un livre qui peut servir à la connaissance de soi-même ; en rentrant de la campagne je me suis prescrit de lire chaque jour cinq chapitres de l'Evangile ; parce qu'étant ici je veux absolument lire tout le nouveau Testament, une fois en russe et une fois en slavon ; j'aime la langue de mon pays et je veux la maîtriser ; et j'ai du plaisir à lire l'Evangile en ma langue $]^{37}$.

Le désir de «maîtriser » le russe (littéralement : «s'affermir » dans cette langue) est évidemment lié à un certain affermissement de soi, capable d'adopter, à travers une autre langue, un autre point de vue. Elizaveta Šahovskaja abandonne les formules françaises, coulantes et faciles, pour un autre mode d'expression, plus difficile pour elle, mais plus « direct» et « authentique », qui lui permet une plus

33. Elle a pu lire une édition récente : Le combat spirituel, par le R.P.D. Laurent Scrupoli, suivi d'un Traité de la paix de l'âme, par le même auteur, traduction nouvelle par ***, augmentée de prières tirées des Paraphrases de Massillon, et d'un morceau inédit du P. Bourdaloue, P. : Librairie grecque-latine-allemande, 1820.

34. L'Évangile médité et distribué pour tous les jours de l'année, œuvre du jésuite Bonaventure Giraudeau, parachevée et publiée par l'abbé Arnaud Duquesne en 1773-1774, et qui connut de nombreuses rééditions.

35. E.A. Šahovskaja, [Journal], RGB, fonds 336/2, carton 47, $n^{\circ} 5, f^{\circ} 4$.

36. Ibid., $\mathrm{n}^{\circ} 3, \mathrm{f}^{\circ} 19$.

37. Ibid., $\mathrm{n}^{\circ} 2, \mathrm{f}^{\circ} 132 \mathrm{v}^{\circ}$. 
grande individualisation. Elle passe souvent au russe dans les moments « décisifs » de l'épanchement émotionnel, de la mise en scène de soi et de ses luttes intérieures :

Mais je me laisse guider par mon imagination. И я все придумала для будущего счастия, а ничего для того, что мне делать и как поступать, когда ты возворотишься. Ты не поверишь, как трудно быть или лутче сказать казаться чужой для такого человека, котораго любишь более всех [Et j'ai pensé à tout ce qui a rapport au bonheur futur, mais je ne sais que faire et comment me conduire quand tu reviendras. Tu ne croiras pas combien il est difficile d'être, ou plutôt de paraître étrangère pour celui que l'on aime le plus]. Reviens mon ami, et pense à ce que nous devons faire.... ${ }^{38}$

Elle tente aussi d'exprimer en russe son sentiment d'amour, ce qui semble lui être particulièrement délicat :

Je t'attends, mon ami, viendras-tu ? Je suis très disposée à te faire part de mes sentiments. Я право не знаю, от чего я чувствую такое особенное и неизъяснимое чувство. Я всегда его люблю, а сегодня мне мало его любить, мне нужно ему изъяснить, как много я его люблю [Je ne sais vraiment pourquoi j'éprouve un sentiment si spécial et indicible. Je l'aime toujours, et aujourd'hui cela ne me suffit pas de l'aimer, je dois encore lui dire, combien je l'aime]. En dépit des convenances qui ne mettent que des entraves aux jouissances réelles, je lui dirai que je l'adore. ${ }^{39}$

Le russe est ainsi choisi pour accentuer la gravité de son discours amoureux qui acquiert, grâce à ce choix linguistique, des accents à la fois fermes et fiers :

Нет, я до тех пор не отвергну надежду, как ты мне сам скажешь, что ты меня более не любишь, что «я тебе чужая», но, друг мой, я надеюсь,что ты не переменился - C тех пор как мы знаем друг друга, мы не переставали любить, иногда теряли один другого из виду, или лучше сказать были отвлекаемы, теми людьми, которым приятно разлучать... [Non, je ne cesserai d'espérer avant que tu ne me dises que tu ne m'aimes plus, que « je te suis etrangère », mais mon ami, j'espère que tu n'as pas changé - Depuis que nous nous connaissons, nous n'avons pas cessé de nous aimer, parfois nous nous perdions l'un l'autre de vue, ou plutôt, nous étions distraits par ceux qui aiment séparer... $]^{40}$

Marija Tolstaja - jeune fille restée orpheline et qui, à l'époque de la rédaction de son journal de 1821-1823 tenu à Pétersbourg et à Carskoe Selo, se trouve sous la tutelle de sa tante, demoiselle d'honneur de chambre de l'impératrice Elisabeth Alekseevna - se réjouit dans son journal de pouvoir parler sa langue maternelle «du moins » avec sa cousine à qui elle adresse ses notes. Elle choisit le russe lorsqu'elle veut restituer son monde à elle, se distancier de la société mondaine - y

38. Ibid., $\mathrm{f}^{\circ} 20$.

39. Ibid., $\mathrm{n}^{\circ} 3, \mathrm{f}^{\circ} 1 \mathrm{v}^{\circ}$.

40. Ibid., $\mathrm{n}^{\circ} 2, \mathrm{f}^{\circ} 12$. 
compris au moyen de l' «écran » que constitue la langue populaire - , mettre en relief sa propre personnalité :

Il pleut à verse ; il fait humide ; j'ai dîné avec madame Pitt ${ }^{41}$ qui avait son fils, et un autre jeune homme anglais, un cousin de son fils, et puis donc Constance. Bce англичаны, а не руския - у них все свой разговор, про Лондон, англичан, для меня они все чужие, а их знакомые для меня не интересны. О! как сердце мое желает быть с своими. [...] Чай нада итти мне опять к madame Pitt, нада быть веселой, а то скажут, что за прескучная княжна Оболенская; а сердце что то не лежит к радости. [...] Я рада хоть с тобой на природном языке поговорить... [Tous sont Anglais et non pas Russes - ils ont leur conversation à eux, ils parlent de Londres, des Anglais, ils sont tous des étrangers pour moi, et leur entourage ne m'est pas intéressant. Oh, combien mon cœur veut être avec les siens. [...] Il faut peut-être de nouveau aller chez madame Pitt, il faut être joyeuse, sinon on dira : que la princesse Obolenskaja est ennuyeuse ; mais mon cœur n'est pas disposé à la joie, je ne sais pourquoi. [...] Je me rejouis de parler ma langue naturelle au moins avec toi...]. ${ }^{42}$

Le français cède la place au russe lors de la prise de conscience de son expérience individuelle ; pour Marija Tolstaja, c'est son état d'orpheline qui détermine sa vision du monde et ses rapports avec son entourage :

Je me suis assise sur un canapé toute seule, songeant, combien il était terrible d'être orpheline, когда некому заступиться [quand il n'y a personne pour vous défendre], il faut souffrir ; Je ne suis pas bien depuis trois ou quatre jours, je ne sais ce que j'ai, mais j'ai un malaise, je suis pâle, pâle comme la mort, et mon mal de poitrine se fait sentir par ci, par là - скучно, скучно, Варинька! Ну чтой то, ей Богу, вечно под властью тридцати нянек быть... [que c'est triste, triste, Varin'ka, d'être éternellement, comme un enfant, gouverné par trente bonnes... $]^{43}$.

Le russe sert aussi à traduire les émotions fortes dans le journal de 1821 d'Ekaterina Ivanovna Vadkovskaja (née comtesse Černyševa ; 1765-après 1826), qu'elle tient à un âge avancé, après avoir passé sa jeunesse à la cour de Catherine II :

Mon Dieu ! Qu'il m'est fatiguant de vivre, так тяжело нести жизнь, тащить, тащить еe! Ox! Куда как тяжело! Мочи нету, устала [c'est si difficile de traîner sa vie ! Oh ! Comme c'est difficile ! Je n'en puis plus, je suis fatiguée] ${ }^{44}$.

Le russe des diaristes inclut parfois des gallicismes, inévitables à cette époque : « Я всегда его люблю » [je l'aime toujours], «мы [...] были отвлекаемы » [nous étions distraits], « я не имею ничего нового сказать тебе » [je n'ai rien de neuf à te dire $]^{45}$, écrit Elizaveta Šahovskaja; «буду [...] говорить их древний

41. Lectrice de l'impératrice Elisabeth Aleksejevna.

42. « Si tu lis jamais ce journal... », p. 252-253.

43. M.J. Tolstaja, [Journal] (autographe), RGADA, f. 1280, op. 1, d. 134, 1. 37, 81 .

44. E.I. Vadkovskaja, [Journal] (autographe), RGALI (Rossijskij gosudarstvennyj arhiv literatury i iskusstva - Archives d'État de Russie de littérature et d'art), f. 1337, op. 3, d. 18,1. 7.

45. « Si tu lis jamais ce journal... », p. 234-235. 
шотландский язык » [je vais parler leur ancienne langue écossaise], écrit Marija Tolstaja à propos de sa lecture d'un roman de Walter Scott ${ }^{46}$.

Mais leur russe est surtout proche de la langue du peuple avec ses tournures et ses expressions idiomatiques, de cette langue dont se distanciaient Karamzin et ses adeptes : «Так иной раз так скучно, что хоть плакать » [C'est si triste parfois, triste à pleurer] ; « сердце что то не лежит к радости » [mon cœur n'est pas disposé à la joie]; «Я тебе надоела, не знаю как тебя оставить, хоть я из пустова в порожнее переливаю » [Tu en as assez de moi, mais je ne sais comment te laisser, quoique je ne fasse que rabâcher ${ }^{47}$; «когда хожу, не разбираю дороги » [quand je marche, je ne vois pas le chemin] ${ }^{48}$.

Ces tournures alternent et s'amalgament parfois avec celles du français: «combien il était terrible d'être orpheline, когда некому заступиться », « Валентина так и подмывает танцовать [Valentin est tout tenté de danser], et en même tems il craint l'impression que j'en aurai ${ }^{49}$, «pour noter notre житиебытие »[existence $]^{50}$.

Une parfaite maîtrise du français, tel qu'il était formé par la culture salonnière et la littérature du XVIII siècle et, en même temps, la pratique du russe populaire est un des traits caractéristiques du langage de la noblesse à cette époque, qui fit souvent l'objet de représentations caricaturales ${ }^{51}$. Or, à en juger notamment d'après les textes de nos diaristes, cette particularité mérite plutôt d'être remise en valeur. La maîtrise de différents modes d'expression à travers les deux langues, le passage immédiat d'un registre à l'autre offrent la possibilité de s'orienter librement à travers plusieurs pratiques culturelles, tout en maintenant une certaine distance par rapport à ces pratiques, et en sauvegardant l'espace culturel traditionnel.

elgretchanaia@rambler.ru

IMLI

Académie des Sciences, Moscou

46. Ibid., p. 253.

47. M.J. Tolstaja, [Journal], $\mathrm{f}^{\circ} 73$.

48. E.A. Šahovskaja, [Journal], $\mathrm{n}^{\circ} 2, \mathrm{f}^{\circ} 133 \mathrm{v}^{\circ}$.

49. Ibid., $\mathrm{n}^{\circ} 6, \mathrm{f}^{\circ} 15$.

50. Ibid., $\mathrm{n}^{\circ} 5, \mathrm{f}^{\circ} 26$.

51. V.V. Vinogradov, Očerki russkogo literaturnogo jazyka, p. 223-227. 